\title{
Efficacy and safety of canagliflozin in type 2 diabetes mellitus patients with biopsy-proven nonalcoholic steatohepatitis classified as stage I-3 fibrosis
}

This article was published in the following Dove Press journal: Diabetes, Metabolic Syndrome and Obesity:Targets and Therapy

\author{
Yuya Seko' \\ Taichiro Nishikawa' \\ Atsushi Umemura' \\ Kanji Yamaguchi' \\ Michihisa Moriguchi' \\ Kohichiroh Yasui' \\ Mayumi Kimura ${ }^{2}$ \\ Hiroaki lijima ${ }^{3}$ \\ Toshio Hashimoto ${ }^{3}$ \\ Yoshio Sumida ${ }^{4}$ \\ Takeshi Okanoue ${ }^{5}$ \\ Yoshito Itoh' \\ 'Department of Gastroenterology \\ and Hepatology, Kyoto Prefectural \\ University of Medicine, Kyoto, \\ Japan; ${ }^{2}$ lkuyaku. Integrated Value \\ Development Division, Mitsubishi \\ Tanabe Pharma Corporation, Osaka, \\ Japan; ${ }^{3}$ kuyaku. Integrated Value \\ Development Division, Mitsubishi \\ Tanabe Pharma Corporation, Tokyo, \\ Japan; ${ }^{4}$ Division of Hepatology \\ and Pancreatology, Department of \\ Internal Medicine, Aichi Medical \\ University, Aichi, Japan; ${ }^{5}$ Department \\ of Gastroenterology and Hepatology, \\ Saiseikai Suita Hospital, Osaka, Japan
}

Correspondence: Taichiro Nishikawa Department of Gastroenterology and Hepatology, Kyoto Prefectural University of Medicine, 465 Kajii-cho, KawaramachiHirokoji, Kamigyo-ku, Kyoto 602-8566, Japan

Tel +81752515519

Fax +81752510710

Email taichi@koto.kpu-m.ac.jp

\begin{abstract}
Aim: Nonalcoholic fatty liver disease (NAFLD), including nonalcoholic steatohepatitis (NASH), is known to be associated with type 2 diabetes mellitus (T2DM) in high rate. The improvement in hepatic function due to sodium-glucose co-transporter 2 (SGLT2) inhibitors has been reported in T2DM patients with and without NAFLD. However, only a few studies have attempted to evaluate the role of SGLT2 inhibitors in T2DM patients with biopsy-proven NASH, and no detailed prospective studies including the individual hepatic fibrosis stage have been reported. Therefore, we investigated the effect of canagliflozin on hepatic function in T2DM patients with biopsy-confirmed NASH.
\end{abstract}

Methods: T2DM patients with NASH (hepatic fibrosis stage 1-3 confirmed via liver biopsy, $\mathrm{n}=10$ ) were enrolled and received canagliflozin ( $100 \mathrm{mg}$ ) once a day for 12 weeks. The primary end point was change in serum alanine aminotransferase (ALT) levels from baseline to week 12 . Secondary end points were liver function/fibrosis markers, metabolic parameters, and safety. Results: The change in ALT from baseline to week 12 was $-23.9 \mathrm{U} / \mathrm{L}$ (95\% CI -48.1 to 0.3 , $P=0.0526$ ). Significant improvements in several hepatic function/fibrosis markers, such as aspartate aminotransferase, fibrosis- 4 index, and FM-fibro index, and metabolic parameters including hemoglobin A1c and body weight were found. No serious or liver-related adverse events were reported. Regarding individual patients, different trends in ALT-lowering effects between stage 1 and stage $2 / 3$ subjects were observed; the degree of ALT-lowering effect tended to be greater in the stage 1 group than in the stage $2 / 3$ group.

Conclusion: Our results suggest that canagliflozin is effective and well-tolerated in patients with T2DM and NASH. Canagliflozin may be useful for the treatment of T2DM patients with NASH, especially those in early stages of NASH.

Keywords: canagliflozin, Japanese, NASH, fibrosis stages, SGLT2 inhibitor, type 2 diabetes mellitus

\section{Introduction}

Nonalcoholic fatty liver disease (NAFLD), including nonalcoholic steatohepatitis (NASH), is associated with type 2 diabetes mellitus (T2DM) ${ }^{1-3}$ In Japan, the prevalence of T2DM is $\sim 50 \%$ in patients with NAFLD and increases with progression of the fibrosis stage; DM is a significant risk factor for advanced fibrosis. ${ }^{4}$

Advanced NASH increases the risks of cirrhosis and hepatocellular carcinoma. ${ }^{3}$ A reduction in serum alanine aminotransferase (ALT) level ( $\geq 30 \%$ reduction from the baseline $\mathrm{s}^{5}$ or $\geq 30 \%$ reduction from the baseline and decrease to $\leq 40 \mathrm{U} / \mathrm{L}^{6}$ ) was 
associated with amelioration of liver fibrosis progression in NASH patients. Therefore, control of serum ALT via suitable interventions is important to prevent NASH progression. Although pioglitazone has been shown to improve serum ALT levels and histological features in NASH patients with insulin resistance, concerns such as body weight gain and congestive heart failure exist. ${ }^{7}$ Therefore, new NASH treatment strategies are needed.

Sodium-glucose co-transporter 2 (SGLT2) inhibitors suppress glucose reabsorption in the renal tubules and exert antihyperglycemic effects. In addition to glucose lowering, SGLT2 inhibitors improve multiple risk factors such as body weight and blood pressure..$^{8-10}$ Because some SGLT2 inhibitors including canagliflozin and empagliflozin have shown cardiovascular and renal protective effects in T2DM patients with a history or high risk of cardiovascular disease, ${ }^{11,12}$ they are a potential therapeutic option for preventing diabetic complications. Improvement in hepatic function due to SGLT2 inhibitors has also been reported in T2DM patients with and without NAFLD. ${ }^{13-18}$ We also previously reported that treatment of canagliflozin $(100 \mathrm{mg})$ for 12 weeks significantly improved ALT levels compared with placebo in T2DM patients with ALT >31 U/L. ${ }^{19}$ However, studies on the role of SGLT2 inhibitors in T2DM patients with biopsyproven NASH are limited, and no detailed prospective studies including individual hepatic fibrosis stage data have been reported. ${ }^{16,20,21}$ Therefore, we performed the pilot study to investigate the efficacy and safety of canagliflozin in T2DM patients with biopsy-confirmed NASH.

\section{Methods}

\section{Study design and patients}

A single-arm, exploratory study was performed to investigate the efficacy and safety of canagliflozin in T2DM patients with NASH. The inclusion criteria were as follows: T2DM patients with hemoglobin A $1 \mathrm{c}$ (HbA1c) $\geq 6.0 \%$ and $<10.0 \%$, ALT $\geq 31 \mathrm{U} / \mathrm{L}$, age $\geq 20$ and $<75$ years, hepatic fibrosis stage $1-3^{22,23}$ confirmed via liver biopsies during 1 year prior to the start of the study, and daily alcohol consumption of $<30 \mathrm{~g} /$ day in males and $<20 \mathrm{~g} /$ day in females. The exclusion criteria were as follows: T1DM, history of class IV heart failure per the New York Heart Association functional classification, malignancy, platelet count $<10^{5} / \mu \mathrm{L}$, estimated glomerular filtration rate $<45 \mathrm{~mL} / \mathrm{min} / 1.73 \mathrm{~m}^{2}$, and SGLT2 inhibitor treatment within 12 weeks prior to the start of the study. The severity of hepatic fibrosis stage was scored as: stage 1, zone 3 perisinusoidal fibrosis; stage 2, zone 3 perisinusoidal fibrosis with portal fibrosis; stage 3 , zone 3 perisinusoidal fibrosis and portal fibrosis with bridging fibrosis; and stage 4 , cirrhosis. ${ }^{22,23}$

\section{Intervention and outcomes}

Canagliflozin (100 mg) was orally administered to patients before breakfast for 12 weeks. Visits were scheduled every 4 weeks during the treatment. The primary end point was change in serum ALT levels from baseline. Secondary end points included changes from baseline to week 12 in aspartate aminotransferase (AST), $\gamma$-glutamyl transferase $(\gamma$-GTP), alkaline phosphatase (ALP), hyaluronic acid, type IV collagen 7S, waist circumference, NAFIC score, fibrosis-4 index (FIB-4 index), FM-fibro index calculated from hyaluronic acid and type IV collagen $7 \mathrm{~S},{ }^{24}$ liver stiffness measurement (LSM) and controlled attenuation parameter (CAP) measured via transient elastography (FibroScan; ECHOSENS, Paris, France), HbA1c, fasting plasma glucose, fasting serum insulin, serum C-peptide, platelet count, uric acid, total cholesterol, triglyceride, high-density lipoprotein cholesterol, low-density lipoprotein cholesterol, body composition measured using a multifrequency impedance body composition analyzer (InBody 720; InBody Japan, Tokyo, Japan), and responses to the Dutch Eating Behavior Questionnaire (DEBQ) and to the 8-Item Short Form Health Survey (SF-8). Exploratory end points included changes in other parameters including serum glucagon and ferritin. The safety evaluation included assessments of adverse events (AEs) described using the Medical Dictionary for Regulatory Activities/Japanese version 20.1 and laboratory values.

\section{Sample size calculation}

We assumed a mean difference of $-10 \mathrm{U} / \mathrm{L}$ in the change in ALT levels from baseline to the end of the treatment period with a SD of $15 \mathrm{U} / \mathrm{L}$ based on results from a previous study ${ }^{19}$ and found that 20 patients were required for a power (paired $t$-test) of $81 \%$ at a two-sided significance level of $5 \%$.

\section{Statistical analyses}

Data were represented as mean values with SD or $95 \%$ CI values. All changes between baseline and week 12 were analyzed using two-tailed paired $t$-tests. As subsidiary evaluations, we also analyzed changes after 4 and 8 weeks. A $P$-value $<0.05$ was considered statistically significant. All statistical analyses were performed using the statistical analysis system version 9.4 (SAS Institute Inc., Cary, NC, USA). Data management and statistical analyses were conducted by the EP-CRSU Co. Ltd. (Tokyo, Japan). 


\section{Ethical considerations}

The study was conducted in compliance with the Declaration of Helsinki and the "Ethical guidelines for medical and health research involving human subjects". ${ }^{25}$ It was approved by the institutional review board at Kyoto Prefectural University of Medicine and is registered in the University Hospital Medical Information Network Clinical Trials Registry (UMIN000023044). All patients provided written informed consent.

\section{Results \\ Patients}

Ten patients (seven males and three females) were enrolled in the study and included in efficacy and safety analysis. Patient characteristics are shown in Table 1 . The mean \pm SD of the baseline ALT, HbA1c, and body mass index were $99.1 \pm 52.6$ $\mathrm{U} / \mathrm{L}, 7.09 \pm 0.87 \%$, and $29.09 \pm 2.50 \mathrm{~kg} / \mathrm{m}^{2}$, respectively; age and duration of T2DM were $54.9 \pm 12.9$ and $4.64 \pm 4.38$ years, respectively. Fibrosis stages included stage $1(n=5)$, stage 2 $(n=3)$, and stage $3(n=2)$.

Table I Patient demographics and characteristics

\begin{tabular}{|c|c|}
\hline & $\mathbf{N}$ or mean (SD) \\
\hline \multicolumn{2}{|l|}{ Sex } \\
\hline Male & 7 \\
\hline Female & 3 \\
\hline Age (years) & $54.9(12.9)$ \\
\hline Duration of diabetes (years) & $4.64(4.38)$ \\
\hline $\mathrm{HbAlc} \mathrm{( \% )}$ & $7.09(0.87)$ \\
\hline Fasting plasma glucose (mg/dL) & $126.9(29.8)$ \\
\hline HOMA-IR & $4.10(1.74)$ \\
\hline Body weight (kg) & $77.5 \mathrm{I}(12.07)$ \\
\hline $\mathrm{BMI}\left(\mathrm{kg} / \mathrm{m}^{2}\right)$ & $29.09(2.50)$ \\
\hline $\mathrm{ALT}(\mathrm{U} / \mathrm{L})$ & 99.1 (52.6) \\
\hline AST (U/L) & $69.9(37.8)$ \\
\hline$\gamma$-GTP $(\mathrm{U} / \mathrm{L})$ & $73.7(41.1)$ \\
\hline Platelet $\left(\times 10^{3} / \mu \mathrm{L}\right)$ & $216.9(67.7)$ \\
\hline eGFR $\left(\mathrm{mL} / \mathrm{min} / \mathrm{I} .73 \mathrm{~m}^{2}\right)$ & $79.1(16.0)$ \\
\hline \multicolumn{2}{|l|}{ Complications } \\
\hline Diabetic retinopathy & I \\
\hline Hypertension & 6 \\
\hline Dyslipidemia & 8 \\
\hline \multicolumn{2}{|l|}{ Concomitant antidiabetic drugs } \\
\hline No & 5 \\
\hline Yes & 5 \\
\hline \multicolumn{2}{|l|}{ Fibrosis stage } \\
\hline Stage I & 5 \\
\hline Stage 2 & 3 \\
\hline Stage 3 & 2 \\
\hline
\end{tabular}

Abbreviations: ALT, alanine aminotransferase; AST, aspartate aminotransferase; BMI, body mass index; eGFR, estimated glomerular filtration rate; $\gamma$-GTP, $\gamma$-glutamyl transpeptidase; HbAlc, hemoglobin Alc; HOMA-IR, homeostasis model of assessment-insulin resistance.

\section{Efficacy}

A decreasing trend in ALT levels was observed from week 4 (Figure 1A), and the mean change in ALT levels from baseline to week 12 (primary end point) was $-23.9 \mathrm{U} / \mathrm{L}$ (95\% CI -48.1 to $0.3, P=0.0526$ ) (Table 2), corresponding to the percent change of $-19.94 \%$ (95\% CI -40.40 to 0.53$)$. AST, HbA1c, and body weight decreased from week 4 and were significantly reduced at week 12 (Figure 1B-D) (Table 2).

The other secondary end points, $\gamma$-GTP as a hepatic function-related marker, and platelets, type IV collagen 7S, the FIB-4 index and the FM-fibro index as hepatic fibrosis markers, were significantly changed at week 12 . Other hepatic fibrosis markers including the NAFIC score and LSM/CAP evaluated using transient elastography did not change (Table 2).

Changes in body composition were assessed using a multifrequency impedance body composition analyzer; body fat mass was significantly decreased, and no significant changes in muscle mass or body water content were seen (Table 2). The percentage changes in each component are shown in Table S1.

Changes in ALT in each subject are shown in Figure 2; different trends in ALT-lowering effects between stage 1 and stage $2 / 3$ subjects were observed. Post hoc analysis showed that in the stage 1 and stage $2 / 3$ populations, the baseline ALT values were $109.6 \pm 59.7$ and $88.6 \pm 49.0 \mathrm{U} / \mathrm{L}$, respectively; changes in the ALT values from baseline to week 12 were $-46.2 \mathrm{U} / \mathrm{L}(95 \% \mathrm{CI}-84.0$ to -8.4$)$ and $-1.6 \mathrm{U} / \mathrm{L}(95 \% \mathrm{CI}$ -26.8 to 23.6 ), respectively.

Other secondary end points and exploratory end points are shown in Table S2. Among them, serum ferritin was significantly decreased at week 12 . The SF-8 and DEBQ, indicators of quality of life and dietary habits, respectively, were unchanged due to the treatment (data not shown).

\section{Safety}

AEs occurred in five patients; however, no serious or liverrelated AEs were reported (Table S3). The changes in laboratory values are shown in Table S4.

\section{Discussion}

This study examined the efficacy and safety of canagliflozin in T2DM patients with NASH; the treatment showed improvement in several hepatic function/fibrosis markers and metabolic parameters and was well-tolerated. Canagliflozin decreased ALT levels from baseline (-23.9 U/L); however, it was not statistically significant $(P=0.0526)$. 
A

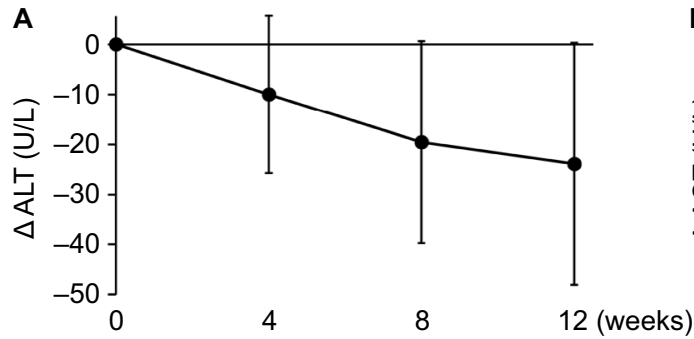

C

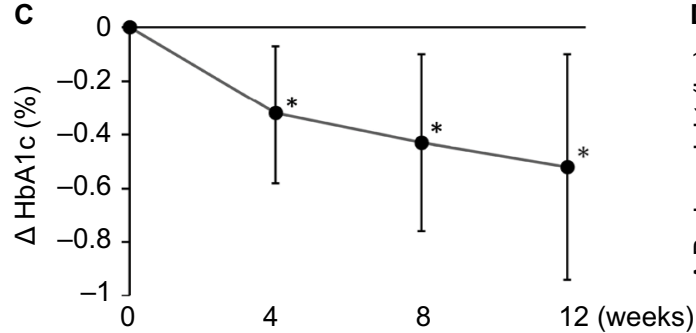

B

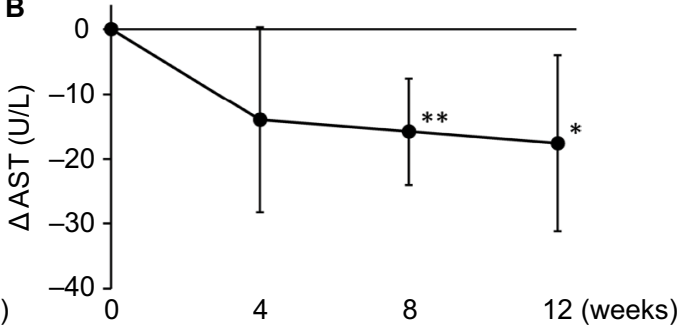

Figure I Changes in (A) ALT, (B) AST, (C) HbAIc, and (D) body weight over 12 weeks.

Note: $* P<0.05, * * P<0.0$ I vs baseline (paired $t$-test).

Abbreviations: ALT, alanine aminotransferase; AST, aspartate aminotransferase; HbAlc, hemoglobin Alc.

Table 2 Effects of canagliflozin on hepatic function/fibrosis markers and metabolic parameters $(n=10)$

\begin{tabular}{|c|c|c|c|c|c|}
\hline & \multirow{2}{*}{$\begin{array}{l}\text { Week 0, } \\
\text { Mean (SD) }\end{array}$} & \multirow{2}{*}{$\begin{array}{l}\text { Week I2, } \\
\text { Mean (SD) }\end{array}$} & \multicolumn{2}{|c|}{ Changes from week 0} & \multirow{2}{*}{$\begin{array}{l}\text { Paired } t \text {-test } \\
\text { (P-value) }\end{array}$} \\
\hline & & & Mean (SD) & $95 \% \mathrm{Cl}$ & \\
\hline ALT (U/L) & $99.1(52.6)$ & $75.2(39.7)$ & $-23.9(33.9)$ & $(-48.1$ to 0.3$)$ & 0.0526 \\
\hline AST (U/L) & $69.9(37.8)$ & $52.3(26.2)$ & $-17.6(19.0)$ & $(-31.2$ to -4.0$)$ & 0.0168 \\
\hline$\gamma-\mathrm{GTP}(\mathrm{U} / \mathrm{L})$ & $73.7(4 I .1)$ & $57.7(30.3)$ & $-16.0(15.4)$ & $(-27.0$ to -5.0$)$ & 0.0094 \\
\hline Platelet $\left(10^{3} / \mu \mathrm{L}\right)$ & $216.9(67.7)$ & $240.3(74.0)$ & $23.4(21.3)$ & (8.I to 38.7$)$ & 0.0071 \\
\hline Type IV collagen 7S (ng/mL) & $5.72(1.36)$ & $5.13(1.06)$ & $-0.59(0.64)$ & $(-1.05$ to -0.13$)$ & 0.0176 \\
\hline FIB-4 index & $2.077(1.207)$ & $\mathrm{I} .602(0.98 \mathrm{I})$ & $-0.475(0.385)$ & $(-0.750$ to -0.200$)$ & 0.0036 \\
\hline FM-fibro index & $0.545(0.133)$ & $0.491(0.104)$ & $-0.054(0.070)$ & $(-0.104$ to -0.004$)$ & 0.0376 \\
\hline NAFIC score & $2.1(1.4)$ & $1.9(1.5)$ & $-0.2(0.8)$ & $(-0.8$ to 0.4$)$ & 0.4433 \\
\hline $\mathrm{CAP}(\mathrm{dB} / \mathrm{m})$ & $327.4(38.2)$ & $321.4(36.4)$ & $-6.0(56.5)$ & $(-46.4$ to 34.4$)$ & 0.7447 \\
\hline LSM (kPa) & $8.85(4.79)$ & 9.09 (7.39) & $0.24(3.58)$ & $(-2.32$ to 2.80$)$ & 0.8367 \\
\hline HbAlc (\%) & $7.09(0.87)$ & $6.57(0.55)$ & $-0.52(0.59)$ & $(-0.94$ to -0.10$)$ & 0.0218 \\
\hline Fasting plasma glucose (mg/dL) & $126.90(29.85)$ & II5.40 (I5.3I) & $-11.50(18.17)$ & $(-24.50$ to 1.50$)$ & 0.0763 \\
\hline Body weight $(\mathrm{kg})$ & $77.5 \mathrm{I}(12.07)$ & $75.42(12.17)$ & $-2.09(1.87)$ & $(-3.43$ to -0.75$)$ & 0.0064 \\
\hline Body fat mass (kg) & $26.25(4.80)$ & $24.60(4.93)$ & $-1.65(1.65)$ & $(-2.83$ to -0.47$)$ & 0.0116 \\
\hline Skeletal muscle mass (kg) & $28.4 \mathrm{I}(6.92)$ & $28.21(7.10)$ & $-0.20(0.57)$ & $(-0.6 \mathrm{I}$ to $0.2 \mathrm{I})$ & 0.2972 \\
\hline Total body water (L) & $37.76(8.23)$ & $37.43(8.42)$ & $-0.33(0.82)$ & $(-0.92$ to 0.26$)$ & 0.2339 \\
\hline BMI $\left(\mathrm{kg} / \mathrm{m}^{2}\right)$ & $29.09(2.50)$ & $28.28(2.55)$ & $-0.81(0.70)$ & $(-1.3 \mid$ to $-0.3 \mid)$ & 0.0052 \\
\hline Uric acid (mg/dL) & $6.15(1.27)$ & $5.09(0.90)$ & $-1.06(0.75)$ & $(-1.60$ to -0.52$)$ & 0.0016 \\
\hline
\end{tabular}

Note: The laboratory reference range of each parameters: ALT (male) 10-42 U/L, ALT (female) 7-23 U/L, AST (male and female) I3-30 U/L; $\gamma$-GTP (male) I3-64 U/L, $\gamma$-GTP (female) 9-32 U/L.

Abbreviations: ALT, alanine aminotransferase; AST, aspartate aminotransferase; BMI, body mass index; CAP, controlled attenuation parameter; FIB-4 index, fibrosis-4 index; $\gamma$-GTP, $\gamma$-glutamyl transpeptidase; HbAIc, hemoglobin AIc; LSM, liver stiffness measurement.

This may have been due to a lack of statistical power. Canagliflozin significantly improved the hepatic fibrosis markers such as the FIB-4 index and the FM-fibro index, suggesting the possibility of improving hepatic fibrosis.
Conversely, NAFIC score, LSM, and CAP did not change; improvement in these parameters may not be detected during short-term treatments. Improvement in several hepatic function/fibrosis markers (ALT, AST, FIB-4 index) was 


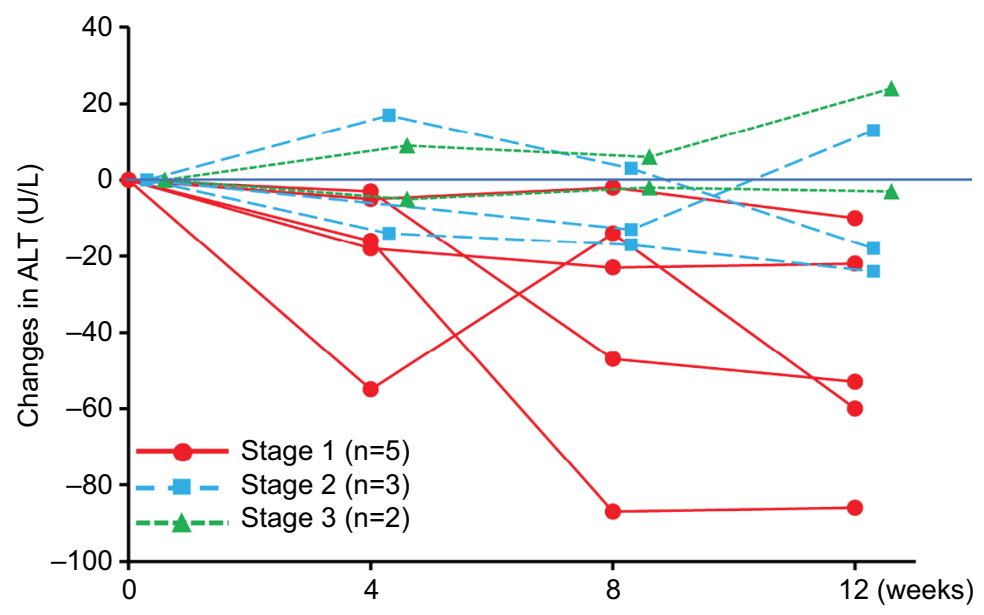

Figure 2 Time course of changes in ALT levels from baseline in individual subjects.

Note: The degree of ALT-lowering effect tended to be greater in the stage I group than in the stage $2 / 3$ group.

Abbreviation: ALT, alanine aminotransferase.

consistent with previous reports involving SGLT2 inhibitors, ${ }^{20,21}$ except the FM-fibro index, a new fibrosis marker, ${ }^{24}$ with the present study being the first reporting its use in drug evaluation. Although accuracy of FM-fibro index for diagnosing NASH fibrosis had been evaluated in a pilot ${ }^{26}$ or multicenter studies, ${ }^{27}$ the drug evaluation data is also important for characterization of FM-fibro index as a biomarker of NASH. In terms of hepatic fibrosis stage, decrease in ALT tended to be greater in the stage 1 group $(-46.2 \mathrm{U} / \mathrm{L})$ than in the stage $2 / 3$ group $(-1.6 \mathrm{U} / \mathrm{L})$, suggesting greater improvement in hepatic function in the stage 1 group; this may indicate the significance of canagliflozin treatment in early-stage NASH patients.

In addition to reduction in ALT, improvement in $\mathrm{HbA} 1 \mathrm{c}^{28}$ and body weight reduction ${ }^{29}$ are also important for preventing NASH progression. Adipose tissue inflammation is also associated with NASH progression ${ }^{30}$; canagliflozin reduced fat mass, which may lead to inhibition of inflammatory cytokine release from adipocytes. ${ }^{31}$ The effects of SGLT2 inhibitors on these factors may contribute to prevention of NASH progression.

NAFLD/NASH with diabetes increases the risk of developing cardiovascular disease and serious hepatic diseases, particularly NASH, cirrhosis, and hepatocellular carcinoma. ${ }^{32,33}$ Moreover, malignant neoplasia is the leading cause of death and liver cancer is the second leading cause of cancer death in Japanese DM patients. ${ }^{34}$ Therefore, interventions using SGLT2 inhibitors in T2DM patients with early-stage NASH may be important to prevent cardiovascular disease and liver cancer.
The limitations of this study were the small sample size, the short-term treatment period, and the lack of a control group.

\section{Conclusion}

Canagliflozin is effective and well-tolerated in patients with T2DM and NASH; canagliflozin may be especially useful for those in early stages of NASH. A long-term, large-scale, randomized control study is necessary to confirm the benefit of SGLT2 inhibitor treatment in patients with T2DM and $\mathrm{NASH}$

\section{Data sharing statement}

The datasets generated and/or analyzed during the current study are not publicly available because of lack of agreement for disclosing individual raw data in public but are available from the corresponding author on reasonable request.

\section{Acknowledgments}

This study was funded by Mitsubishi Tanabe Pharma Corporation. The authors would like to thank Ms A Yano (EP-CRSU Co., Ltd.) for coordinating the study and Ms Y Yamamoto (EP-CRSU Co., Ltd.) for conducting the statistical analyses.

\section{Author contributions}

Y Seko,TN and YI contributed to the study design, data collection and analysis. Y Sumida, TO, MK, HI, and TH contributed to the study design. All authors contributed to data interpretation, drafting or revising the article, gave final approval of the version to be published, and agree to be accountable for all aspects of the work. 


\section{Disclosure}

Y Sumida has received speakers' bureau fees from Astellas Pharma Inc., AstraZeneca K.K., Eli Lilly Japan K.K., MSD K.K., Mitsubishi Tanabe Pharma Corporation, Ono Pharmaceutical Co., Ltd., and Sumitomo Dainippon Pharma Co., Ltd. and has received a research grant from Bristol-Myers Squibb Co. TO has received consulting fee from Bristol-Myers Squibb Co., and Gilead Sciences, Inc. MK, HI, and TH are employees of Mitsubishi Tanabe Pharma Corporation. YI has received speakers' bureau fees from Mitsubishi Tanabe Pharma Corporation and AU has received research support from Bristol-Myers Squibb Co. Y Seko, TN, KY, MM, and KY report no conflicts of interest in this work.

\section{References}

1. Vernon G, Baranova A, Younossi ZM. Systematic review: the epidemiology and natural history of non-alcoholic fatty liver disease and non-alcoholic steatohepatitis in adults. Aliment Pharmacol Ther. 2011;34(3):274-285.

2. Reid AE. Nonalcoholic steatohepatitis. Gastroenterology. 2001; 121(3):710-723.

3. Younossi ZM, Koenig AB, Abdelatif D, Fazel Y, Henry L, Wymer M. Global epidemiology of nonalcoholic fatty liver disease - meta-analytic assessment of prevalence, incidence, and outcomes. Hepatology. 2016;64(1):73-84.

4. Nakahara T, Hyogo H, Yoneda M, et al; Japan Study Group of Nonalcoholic Fatty Liver Disease. Type 2 diabetes mellitus is associated with the fibrosis severity in patients with nonalcoholic fatty liver disease in a large retrospective cohort of Japanese patients. J Gastroenterol. 2014;49(11):1477-1484.

5. Seko Y, Sumida Y, Tanaka S, et al. Serum alanine aminotransferase predicts the histological course of non-alcoholic steatohepatitis in Japanese patients. Hepatol Res. 2015;45(10):E53-E61.

6. Hoofnagle JH, Van Natta ML, Kleiner DE, et al; Non-alcoholic Steatohepatitis Clinical Research Network (NASH CRN). Vitamin E and changes in serum alanine aminotransferase levels in patients with nonalcoholic steatohepatitis. Aliment Pharmacol Ther. 2013;38(2):134-143.

7. Watanabe S, Hashimoto E, Ikejima K, et al; Japanese Society of Gastroenterology; Japan Society of Hepatology. Evidence-based clinical practice guidelines for nonalcoholic fatty liver disease/nonalcoholic steatohepatitis. J Gastroenterol. 2015;50(4):364-377.

8. Kashiwagi A, Maegawa $\mathrm{H}$. Metabolic and hemodynamic effects of sodium-dependent glucose cotransporter 2 inhibitors on cardio-renal protection in the treatment of patients with type 2 diabetes mellitus. $J$ Diabetes Investig. 2017;8(4):416-427.

9. Inagaki N, Harashima SI, Iijima H. Canagliflozin for the treatment of type 2 diabetes: a comparison between Japanese and non-Japanese patients. Expert Opin Pharmacother. 2018;19(8):895-908.

10. Davies MJ, Merton KW, Vijapurkar U, Balis DA, Desai M. Canagliflozin improves risk factors of metabolic syndrome in patients with type 2 diabetes mellitus and metabolic syndrome. Diabetes Metab Syndr Obes. 2017; 10:47-55.

11. Neal B, Perkovic V, Mahaffey KW, et al; CANVAS Program Collaborative Group. Canagliflozin and cardiovascular and renal events in type 2 diabetes. N Engl J Med. 2017;377(7):644-657.

12. Zinman B, Wanner C, Lachin JM, et al; EMPA-REG OUTCOME Investigators. Empagliflozin, cardiovascular outcomes, and mortality in type 2 diabetes. N Engl J Med. 2015;373(22):2117-2128.

13. Leiter LA, Forst T, Polidori D, Balis DA, Xie J, Sha S. Effect of canagliflozin on liver function tests in patients with type 2 diabetes. Diabetes Metab. 2016;42(1):25-32.
14. Ito D, Shimizu S, Inoue $\mathrm{K}$, et al. Comparison of ipragliflozin and pioglitazone effects on nonalcoholic fatty liver disease in patients with type 2 diabetes: a randomized, 24-week, open-label, active-controlled trial. Diabetes Care. 2017;40(10):1364-1372.

15. Shibuya T, Fushimi N, Kawai M, et al. Luseogliflozin improves liver fat deposition compared to metformin in type 2 diabetes patients with non-alcoholic fatty liver disease: a prospective randomized controlled pilot study. Diabetes Obes Metab. 2018;20(2):438-442.

16. Seko Y, Sumida Y, Tanaka S, et al. Effect of sodium glucose cotransporter 2 inhibitor on liver function tests in Japanese patients with nonalcoholic fatty liver disease and type 2 diabetes mellitus. Hepatol Res. 2017;47(10):1072-1078.

17. Kuchay MS, Krishan S, Mishra SK, et al. Effect of empagliflozin on liver fat in patients with type 2 diabetes and nonalcoholic fatty liver disease: a randomized controlled trial (E-LIFT Trial). Diabetes Care. 2018;41(8):1801-1808.

18. Sumida Y, Murotani K, Saito M, et al. Effect of luseogliflozin on hepatic fat content in type 2 diabetes patients with non-alcoholic fatty liver disease: a prospective, single-arm trial (LEAD trial). Hepatol Res. Epub 2018 Jul 27.

19. Seko Y, Sumida Y, Sasaki K, et al. Effects of canagliflozin, an SGLT2 inhibitor, on hepatic function in Japanese patients with type 2 diabetes mellitus: pooled and subgroup analyses of clinical trials. $J$ Gastroenterol. 2018;53(1):140-151.

20. Tobita H, Sato S, Miyake T, Ishihara S, Kinoshita Y. Effects of dapagliflozin on body composition and liver tests in patients with nonalcoholic steatohepatitis associated with type 2 diabetes mellitus: a prospective, open-label, uncontrolled Study. Curr Ther Res Clin Exp. 2017;87:13-19.

21. Akuta N, Watanabe C, Kawamura Y, et al. Effects of a sodium-glucose cotransporter 2 inhibitor in nonalcoholic fatty liver disease complicated by diabetes mellitus: preliminary prospective study based on serial liver biopsies. Hepatol Commun. 2017;1(1):46-52.

22. Brunt EM, Kleiner DE, Wilson LA, Belt P, Neuschwander-Tetri BA; NASH Clinical Research Network (CRN). Nonalcoholic fatty liver disease (NAFLD) activity score and the histopathologic diagnosis in NAFLD: distinct clinicopathologic meanings. Hepatology. 2011;53(3):810-820.

23. Brunt EM, Janney CG, Di Bisceglie AM, Neuschwander-Tetri BA, Bacon BR. Nonalcoholic steatohepatitis: a proposal for grading and staging the histological lesions. Am J Gastroenterol. 1999;94(9):2467-2474.

24. Yoshimura K, Okanoue T, Ebise H, et al. Identification of novel noninvasive markers for diagnosing nonalcoholic steatohepatitis and related fibrosis by data mining. Hepatology. 2016;63(2):462-473.

25. Japanese Ministry of Health, Labor and Welfare. Ethical guidelines for medical and health research involving human subjects. Available from: http://www.mhlw.go.jp/file/06-Seisakujouhou-10600000-Daijinkanboukouseikagakuka/0000080278.pdf. Accessed August 29, 2018.

26. Okanoue T, Ebise H, Kai T, et al. A simple scoring system using type IV collagen 7S and aspartate aminotransferase for diagnosing nonalcoholic steatohepatitis and related fibrosis. $J$ Gastroenterol. 2018;53(1):129-139.

27. Itoh Y, Seko Y, Shima T, et al. Accuracy of non-invasive scoring systems for diagnosing non-alcoholic steatohepatitis-related fibrosis: multicenter validation study. Hepatol Res. Epub 2018 Jul 04.

28. Hamaguchi E, Takamura T, Sakurai M, et al. Histological course of nonalcoholic fatty liver disease in Japanese patients: tight glycemic control, rather than weight reduction, ameliorates liver fibrosis. Diabetes Care. 2010;33(2):284-286.

29. Vilar-Gomez E, Yasells-Garcia A, Martinez-PerezY, et al. Development and validation of a noninvasive prediction model for nonalcoholic steatohepatitis resolution after lifestyle intervention. Hepatology. 2016;63(6): $1875-1887$.

30. Tilg H, Moschen AR. Evolution of inflammation in nonalcoholic fatty liver disease: the multiple parallel hits hypothesis. Hepatology. 2010;52(5):1836-1846.

31. Garvey WT, Van Gaal L, Leiter LA, et al. Effects of canagliflozin versus glimepiride on adipokines and inflammatory biomarkers in type 2 diabetes. Metabolism. 2018;85:32-37. 
32. Cusi K. Treatment of patients with type 2 diabetes and non-alcoholic fatty liver disease: current approaches and future directions. Diabetologia. 2016;59(6):1112-1120.

33. Kawamura Y, Arase Y, Ikeda K, et al. Large-scale long-term followup study of Japanese patients with non-alcoholic fatty liver disease for the onset of hepatocellular carcinoma. Am J Gastroenterol. 2012;107(2):253-261.
34. Nakamura J, Kamiya H, Haneda M, et al. Causes of death in Japanese patients with diabetes based on the results of a survey of 45,708 cases during 2001-2010: Report of the Committee on Causes of Death in Diabetes Mellitus. J Diabetes Investig. 2017;8(3): 397-410. 


\section{Supplementary materials}

Table SI Percent change of body composition after canagliflozin treatment at week $12(n=10)$

\begin{tabular}{llll}
\hline & \multicolumn{2}{l}{$\begin{array}{l}\text { Percent changes from week } \\
\mathbf{0}(\%)\end{array}$} & $\begin{array}{l}\text { Paired } \\
\boldsymbol{t} \text {-test } \\
\text { (P-value) }\end{array}$ \\
\cline { 2 - 4 } & Mean (SD) & $\mathbf{9 5 \% ~ C l}$ & 0.0081 \\
\hline Body weight & $-2.70(2.60)$ & $(-4.57$ to -0.91$)$ & 0.0186 \\
Body fat mass & $-6.50(7.20)$ & $(-11.70$ to -1.38$)$ & 0.2235 \\
Skeletal muscle mass & $-0.90(2.10)$ & $(-2.38$ to 0.64$)$ & 0.235 \\
Total body water & $-1.00(2.30)$ & $(-2.64$ to 0.62$)$ & 0.1950 \\
\hline
\end{tabular}

Table S2 Effects of canagliflozin on other efficacy end points $(n=10)$

\begin{tabular}{|c|c|c|c|c|c|}
\hline & \multirow{2}{*}{$\begin{array}{l}\text { Week 0, } \\
\text { Mean (SD) }\end{array}$} & \multirow{2}{*}{$\begin{array}{l}\text { Week I2, } \\
\text { Mean (SD) }\end{array}$} & \multicolumn{2}{|c|}{ Changes from week 0} & \multirow{2}{*}{$\begin{array}{l}\text { Paired } t \text {-test } \\
\text { (P-value) }\end{array}$} \\
\hline & & & Mean (SD) & $95 \% \mathrm{Cl}$ & \\
\hline \multicolumn{6}{|l|}{ Secondary end points } \\
\hline ALP (U/L) & $251.8(42.2)$ & $243.7(54.3)$ & $-8.1(30.8)$ & $(-30.1$ to 13.9$)$ & 0.4274 \\
\hline Hyaluronic acid (ng/mL) & $76.5(52.0)$ & $67.1(46.6)$ & $-9.4(25.4)$ & $(-27.6$ to 8.8$)$ & 0.2725 \\
\hline Fasting serum insulin $(\mu \mathrm{lU} / \mathrm{mL})$ & I3.675 (6.759) & $14.945(6.626)$ & $1.270(6.380)$ & $(-3.294$ to 5.834$)$ & 0.5447 \\
\hline Serum C-peptide (ng/mL) & $2.615(0.58 I)$ & $2.709(0.859)$ & $0.094(0.769)$ & $(-0.456$ to 0.644$)$ & $0.708 I$ \\
\hline Total cholesterol (mg/dL) & $207.9(31.9)$ & $205.6(34.4)$ & $-2.3(17.0)$ & $(-14.5$ to 9.9$)$ & 0.6792 \\
\hline HDL cholesterol (mg/dL) & $49.6(13.7)$ & $50.5(12.0)$ & $0.9(6.3)$ & $(-3.6$ to 5.4$)$ & 0.6602 \\
\hline LDL cholesterol (mg/dL) & $139.0(33.7)$ & I36.3 (34.3) & $-2.7(15.2)$ & $(-13.6$ to 8.2$)$ & 0.5877 \\
\hline Triglyceride (mg/dL) & $133.0(44.6)$ & $145.3(50.9)$ & $12.3(42.3)$ & $(-18.0$ to 42.6$)$ & 0.3818 \\
\hline Waist circumference $(\mathrm{cm})$ & $98.75(5.91)$ & $96.85(5.82)$ & $-1.90(3.96)$ & $(-4.74$ to 0.94$)$ & 0.1639 \\
\hline \multicolumn{6}{|l|}{ Exploratory end points } \\
\hline Ferritin $(\mathrm{ng} / \mathrm{mL})$ & $270.75(243.54)$ & $157.58(151.90)$ & $-113.17(103.43)$ & $(-187.16$ to -39.18$)$ & 0.0072 \\
\hline Glucagon $(\mathrm{pg} / \mathrm{mL})$ & $200.1(61.9)$ & $255.3(127.9)$ & $55.2(77.0)$ & $(0.1$ to $I 10.3)$ & 0.0496 \\
\hline
\end{tabular}

Abbreviations: ALP, alkaline phosphatase; HDL, high-density lipoprotein; LDL, low-density lipoprotein.

Table S3 Adverse events

\begin{tabular}{ll}
\hline & $\mathbf{n}(\%)$ \\
\hline All adverse events & $5(50.0)$ \\
Gastrointestinal disorders & $\mathrm{I}(10.0)$ \\
Abdominal discomfort & $\mathrm{I}(10.0)$ \\
General disorders and administration site conditions & $2(20.0)$ \\
Facial pain & $\mathrm{I}(10.0)$ \\
Oedema & $\mathrm{I}(10.0)$ \\
Pyrexia & $\mathrm{I}(\mathrm{I} 0.0)$ \\
Renal and urinary disorders & $2(20.0)$ \\
Haematuria & $\mathrm{I}(10.0)$ \\
Nocturia & $\mathrm{I}(10.0)$ \\
Pollakiuria & $\mathrm{I}(10.0)$ \\
Reproductive system and breast disorders & $\mathrm{I}(10.0)$ \\
Pruritus genital & $\mathrm{I}(10.0)$ \\
\hline
\end{tabular}

Abbreviation: $n$, number of patients who experienced adverse events. 
Table S4 Laboratory variables $(n=10)$

\begin{tabular}{|c|c|c|c|}
\hline & Week 0 & Week I 2 & Changes from week 0 \\
\hline $\mathrm{SBP}(\mathrm{mmHg})$ & $14 \mid .0(\mid 5.9)$ & $130.8(6.8)$ & $-10.2(15.7)$ \\
\hline $\mathrm{DBP}(\mathrm{mmHg})$ & $85.8(17.5)$ & $78.1(9.0)$ & $-7.7(12.2)$ \\
\hline $\mathrm{Hb}(\mathrm{g} / \mathrm{dL})$ & $15.13(1.55)$ & $15.84(1.19)$ & $0.71(0.89)$ \\
\hline $\mathrm{Ht}(\%)$ & $44.98(4.46)$ & $47.73(3.35)$ & $2.75(2.93)$ \\
\hline \multicolumn{4}{|l|}{ Blood ketone body fraction } \\
\hline Acetoacetate $(\mu \mathrm{mol} / \mathrm{L})$ & $35.9(20.5)$ & $41.8(29.6)$ & $5.9(27.5)$ \\
\hline 3-Hydroxybutyric acid ( $\mu \mathrm{mol} / \mathrm{L})$ & $69.9(50.6)$ & $81.6(60.2)$ & I I.7 (55.5) \\
\hline Total ketone bodies $(\mu \mathrm{mol} / \mathrm{L})$ & $105.8(70.5)$ & $123.4(88.8)$ & | $7.6(8 \mid .7)$ \\
\hline
\end{tabular}

Note: Data are expressed as mean (SD).

Abbreviations: $\mathrm{Hb}$, hemoglobin; $\mathrm{Ht}$, hematocrit.

Diabetes, Metabolic Syndrome and Obesity: Targets and Therapy is an international, peer-reviewed open-access journal committed to the rapid publication of the latest laboratory and clinical findings in the fields of diabetes, metabolic syndrome and obesity research. Original research, review, case reports, hypothesis formation, expert opinion and commentaries are all considered for publication. The manuscript management system is completely online and includes a very quick and fair peer-review system, which is all easy to use. Visit http://www.dovepress.com/testimonials.php to read real quotes from published authors.

Submit your manuscript here: https://www.dovepress.com/diabetes-metabolic-syndrome-and-obesity-targets-and-therapy-journal 